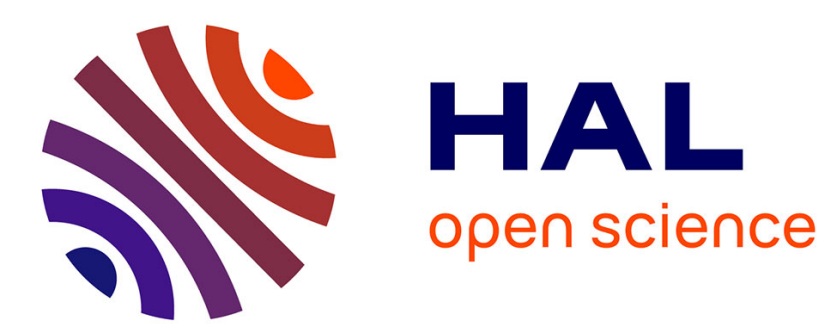

\title{
Spatial Holmboe instability
}

Sabine Ortiz, J.-M. Chomaz, Thomas Loiseleux

\section{To cite this version:}

Sabine Ortiz, J.-M. Chomaz, Thomas Loiseleux. Spatial Holmboe instability. Physics of Fluids, 2002, 14 (8), pp.2585-2597. 10.1063/1.1485078 . hal-00830698

\section{HAL Id: hal-00830698 \\ https://hal-ensta-paris.archives-ouvertes.fr/hal-00830698}

Submitted on 3 Sep 2014

HAL is a multi-disciplinary open access archive for the deposit and dissemination of scientific research documents, whether they are published or not. The documents may come from teaching and research institutions in France or abroad, or from public or private research centers.
L'archive ouverte pluridisciplinaire HAL, est destinée au dépôt et à la diffusion de documents scientifiques de niveau recherche, publiés ou non, émanant des établissements d'enseignement et de recherche français ou étrangers, des laboratoires publics ou privés. 


\section{AIP $\left.\right|^{\text {Physics of }}$ Fluids}

\section{Spatial Holmboe instability}

Sabine Ortiz, Jean-Marc Chomaz, and Thomas Loiseleux

Citation: Physics of Fluids (1994-present) 14, 2585 (2002); doi: 10.1063/1.1485078

View online: http://dx.doi.org/10.1063/1.1485078

View Table of Contents: http://scitation.aip.org/content/aip/journal/pof2/14/8?ver=pdfcov

Published by the AIP Publishing

\section{Articles you may be interested in}

Optimal excitation of two dimensional Holmboe instabilities

Phys. Fluids 23, 074102 (2011); 10.1063/1.3609283

Coupling of Kelvin-Helmholtz instability and buoyancy instability in a thermally laminar plasma

Phys. Plasmas 18, 022110 (2011); 10.1063/1.3555526

Secondary circulations in Holmboe waves

Phys. Fluids 18, 064104 (2006); 10.1063/1.2210504

On Holmboe's instability for smooth shear and density profiles

Phys. Fluids 17, 084103 (2005); 10.1063/1.2001567

Symmetric and nonsymmetric Holmboe instabilities in an inviscid flow

Phys. Fluids 11, 1459 (1999); 10.1063/1.870009

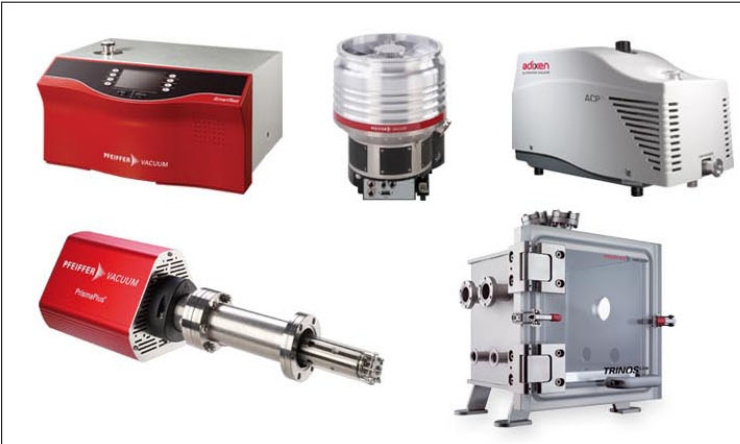

Vacuum Solutions from a Single Source

- Turbopumps

- Backing pumps

- Leak detectors

- Measurement and analysis equipment

- Chambers and components

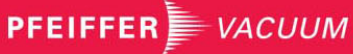




\title{
ARTICLES
}

\section{Spatial Holmboe instability}

\author{
Sabine Ortiz \\ LadHyX, CNRS-Ecole Polytechnique F-91128 Palaiseau Cedex, France \\ and UME/DFA, ENSTA, chemin de la Hunière, F-91761 Palaiseau Cedex, France \\ Jean-Marc Chomaz \\ LadHyX, CNRS-Ecole Polytechnique F-91128 Palaiseau Cedex, France \\ Thomas Loiseleux \\ LadHyX, CNRS-Ecole Polytechnique F-91128 Palaiseau Cedex, France \\ and UME/DFA, ENSTA, chemin de la Hunière, F-91761 Palaiseau Cedex, France
}

(Received 26 February 2001; accepted 19 April 2002; published 18 June 2002)

\begin{abstract}
In mixing-layers between two parallel streams of different densities, shear and gravity effects interplay; buoyancy acts as a restoring force and the Kelvin-Helmholtz mode is known to be stabilized by the stratification. If the density interface is sharp enough, two new instability modes, known as Holmboe modes, appear, propagating in opposite directions. This mechanism has been studied in the temporal instability framework. The present paper analyzes the associated spatial instability problem. It considers, in the Boussinesq approximation, two immiscible inviscid fluids with a piecewise linear broken-line velocity profile. We show how the classical scenario for transition between absolute and convective instability should be modified due to the presence of propagating waves. In the convective region, the spatial theory is relevant and the slowest propagating wave is shown to be the most spatially amplified, as suggested by intuition. Predictions of spatial linear theory are compared with mixing-layer [C. G. Koop and F. K. Browand, J. Fluid Mech. 93, 135 (1979)] and exchange flow [G. Pawlak and L. Armi, J. Fluid Mech. 376, 1 (1999)] experiments. The physical mechanism for Holmboe mode destabilization is analyzed via an asymptotic expansion that predicts the absolute instability domain at large Richardson number.

(c) 2002 American Institute of Physics. [DOI: 10.1063/1.1485078]
\end{abstract}

\section{INTRODUCTION}

Statically stable stratified shear layers have been widely documented due to their interest in geophysical and industrial situations. In the strait of Gibraltar, a stratified shear flow is generated between the heavier hot and salty water exiting the Mediterranean sea and the lighter cold Atlantic water entering into the sea (Armi and Farmer ${ }^{1}$ ). The Strait of Gibraltar is a prominent location where exchange flows naturally exist. A similar flow, called wedge flow, exists in fjords or in estuaries when fresh water enters the ocean space (Pawlak and Armi, ${ }^{2}$ Farmer and Freeland $\left.{ }^{3}\right)$. Stratified shear flows also arise in atmospheric or oceanographic gravity currents resulting from the transient encounter of fluid masses with different densities, e.g., sea or mountain breezes, katabatic winds, ${ }^{4}$ snow avalanches or turbidity currents on the ocean floor $\left(\operatorname{Simpson}^{5}\right)$. In all these situations, instabilities are known to develop at the interface between the different fluid streams and to control the mixing that occurs at the interface. Miles $^{6}$ and Howard ${ }^{7}$ have proven that stability of an inviscid continuous stratified flow is assured if the gradient Richardson number, $R_{i}(y)=-\left[g /\left(\rho(d U / d y)^{2}\right)\right](d \rho / d y)$ [where $g$ represents the gravity, $\rho$ the density, $U(y)$ the velocity as a function of the vertical coordinate $y$ ] which compares locally buoyancy forces to inertia, is everywhere greater than $1 / 4$. This result has been generalized by $\mathrm{Yih}^{8}$ who shows that the same criterion holds when density discontinuities are present. However, Howard and Maslowe ${ }^{9}$ have shown that stratification effects are, in general, more complex since stable stratification adds a restoring force that constrains the vertical displacement of particles, and the instability depends on the details of the velocity and density profiles. A large research effort has been devoted to understanding the effect of buoyancy forces on shear instability by laboratory experiments, ${ }^{10-12}$ numerical studies, ${ }^{13,14}$ and theoretical analysis. ${ }^{15}$ Based upon the totality of these numerical or theoretical studies, which have focused on primarily temporal stability of the stratified shear flows, we know ${ }^{9}$ that if the characteristic thicknesses of the velocity shear and density interface are similar, the instability is stationary with respect to the mean flow, as in homogeneous flow, and is called Kelvin-Helmholtz instability. For a piecewise velocity profile and a two-layer step density model Holmboe ${ }^{16}$ has found theoretically that the shear layer is primarily unstable either to Kelvin-Helmholtz waves or to two traveling waves, one moving upstream and the other downstream with respect to 
the mean speed of the shear layer. In contrast to KelvinHelmholtz modes, those traveling modes called Holmboe modes ${ }^{17}$ in the inviscid approximation, are not restabilized when the stratification increases. Browand and Wang ${ }^{17}$ brought experimental evidence of the existence of Holmboe waves using a stratified water channel. Considering the initial development, they measured the temporal amplification rates and found a fair agreement on the location of the neutral curve predicted by linear theory. ${ }^{16}$ Downstream evolution of the perturbations has been reported by Browand and Winant. ${ }^{10}$ When the bulk Richardson number (value of the gradient Richardson number at $y=0$ ) is low enough, Kelvin-Helmholtz waves develop, roll up and break. When the bulk Richardson number is increased, the roll up is inhibited and the interface starts being deformed by waves traveling in opposite directions, which form peaks protruding into the upper and lower layer. Numerical simulations of a temporally evolving stratified mixing-layer by Smyth, Klassen, and Peltier ${ }^{18}$ have substantiated both the breaking of Kelvin-Helmholtz waves and the development of both traveling waves. Assuming hyperbolic tangent velocity and density profiles, $\mathrm{Hazel}^{13}$ has studied the stability as a function of the ratio of the scale of the shear thickness to the density interface thickness. He shows applying the Miles-Howard criterion $^{6,7}$ that a ratio greater than two is a necessary condition for instability whatever the value of the bulk Richardson number. However, appreciable growth rates for the Holmboe modes are numerically observed for a ratio greater than $2.5 .^{18}$

Previous analysis was focused upon the temporal instability of a stratified shear flow. However, in each field or laboratory situation a reference frame is singled out by boundary conditions and therefore, one should refer to the concept of absolute or convective instability to understand the dynamics of the flow (see Huerre and Monkewitz ${ }^{19}$ for a review). Convectively unstable flows are known to behave as noise amplifiers and their dynamics are described by the spatial theory. In contrast, absolutely unstable flows exhibit selfsustained oscillations even in the absence of external perturbations, since the zero group velocity wave is amplified in the selected frame. For exchange flows, the frame is given by the earth since the flow is in the mean, stationary in this frame. For gravity currents, the selected frame will move with the head of the gravity current that sets up the shear flow. In laboratory mixing-layer facilities, the frame which is singled out is defined by the splitter plate at the end of which the two different fluids streams are set in contact. Studies of the absolute or convective instability in stratified sheared flows are not extensive in the literature and limited to cases where the only primary instability is stationary (i.e., when the shear and density thicknesses are identical). This case has been studied by Lin and Pierrehumbert ${ }^{20}$ and Triantafyllou. ${ }^{21}$ Only recently has the spatial stability theory been addressed by Pawlak and $\mathrm{Armi}^{22}$ in the case of wedge flow where the upper stream is assumed to be at rest, in which the scale of the density stratification is smaller than the scale of the shear and for small bulk Richardson number. They clearly demonstrate that the spatial theory differs strongly from the temporal theory. The most amplified modes are different and the

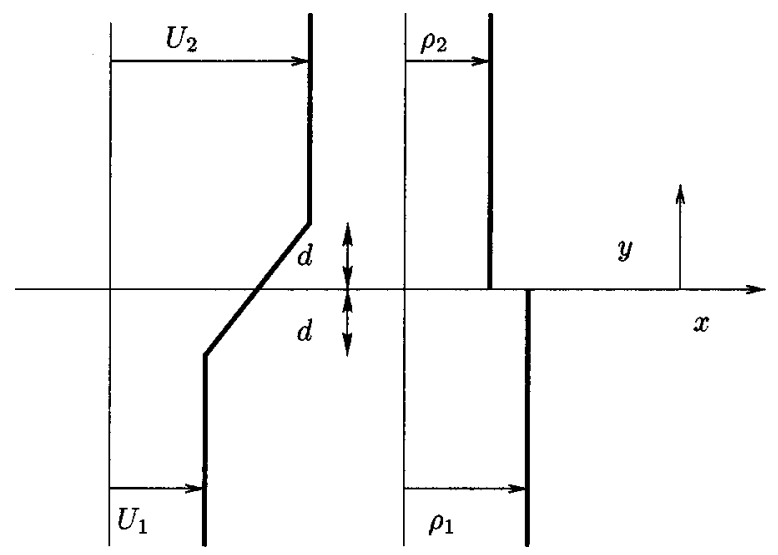

FIG. 1. Velocity and density profiles. An abrupt change in density occurs at the density interface; it is located at $y=0$. Abrupt changes in velocity gradient $d U / d z$ defined the vorticity interfaces located at $y= \pm d$.

spatial instability results from a combination of KelvinHelmholtz and Holmboe instabilities. However, they have addressed neither the effect of the mean advection nor the systematic study of the variations with the Richardson number.

The purpose of the present paper is to extend Pawlak and Armi $^{22}$ analysis to cases where the velocity of both streams are varied independently. Velocities may be in the same direction as in the mixing-layer or in opposite directions as in exchange flows. On a model profile, we will systematically determine the domain where the instability is absolute and where resonances are supposed to occur, varying the mean flow and the bulk Richardson number. This allows us to discuss the absolute and convective transitions for propagating modes. A similar case has been encountered in binary fluid mixtures, ${ }^{23}$ but in the present case the mean advection is not zero and the discussion is more complex than for binary convection. When the instability is convective, spatial growth rates will be determined. We will discriminate between flows where Kelvin-Helmholtz waves are stable and only the two Holmboe waves are unstable (a case not treated by Pawlak and $\mathrm{Armi}^{22}$ ) and cases where the three modes are simultaneously unstable and interact. An asymptotic analysis will allow us to identify the physical mechanism that leads to the destabilization of Holmboe waves and will explain the domain of absolute instability associated to those waves. Finally, we will compare the present theory to field and laboratory experiments and propose some predictions of the present model that might be easily tested in new experiments.

\section{THE MODEL AND THE LINEAR DISPERSION RELATION}

As sketched in Fig. 1, we consider two immiscible, inviscid fluids of constant densities $\rho_{1}$ and $\rho_{2}\left(\rho_{1}>\rho_{2}\right)$ under the Boussinesq approximation (see Drazin and Reid, ${ }^{24}$ p. 35). The layers are considered infinite and surface tension effects are neglected. The index 1 (resp. 2) denotes the lower layer (resp. the upper layer). The dimensionless variables are based on half the vorticity thickness $d$, half the shear inten- 
(a)

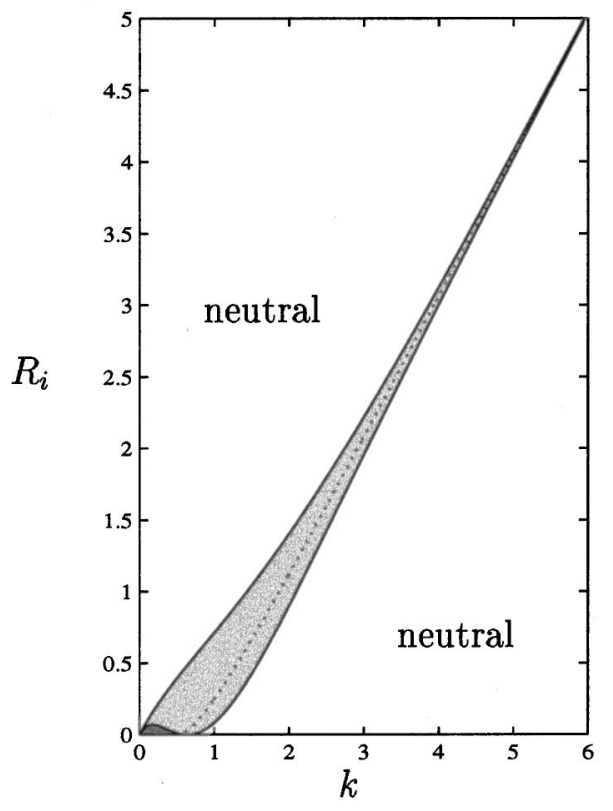

(b)

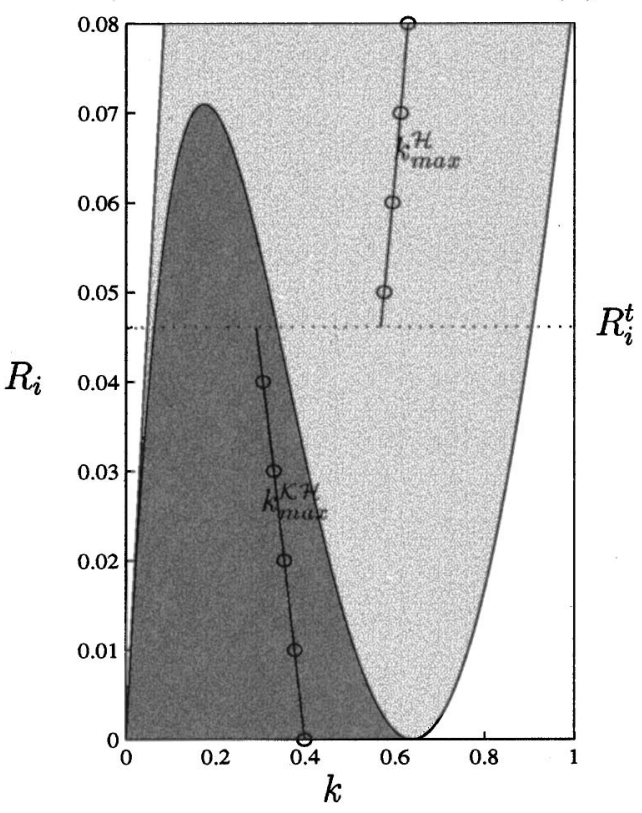

FIG. 2. (a) Stability diagram, (b) zoom of the stability diagram for $R_{i}$ lower than 0.08 . In the dark gray region, Kelvin-Helmholtz modes are unstable, in the light gray region, Holmboe modes are unstable. The asymptotic behavior calculated in (13) is represented by a dotted curve on (a). The locus of the most amplified wave number $k_{\max }$ defined by $\partial \omega_{i} / \partial k\left(k_{\max }\right)=0$ is presented as a solid curve with circles on the zoom (b). The transition between the most amplified Kelvin-Helmholtz mode and the most amplified Holmboe mode is denoted by a dotted line at $R_{i}^{t}$ $=0.0461$. sity $\Delta U=\left(\left|U_{2}-U_{1}\right|\right) / 2$, and the mean density $\left(\rho_{1}+\rho_{2}\right) / 2$. The mean velocity is defined by $U_{m}=\left(U_{1}+U_{2}\right) / 2$. The density interface is located at $y=0$ and the diffusive layer for the stratifying agent (salt or temperature) is supposed infinitely thin for all time (immiscible approximation). In our model, the gradient Richardson number (see Drazin and Reid, ${ }^{24} \mathrm{p}$. $323), R_{i}(y)=-\left[g /\left(\rho(d U / d y)^{2}\right)\right](d \rho / d y)$ has a Dirac function behavior at $y=0$, and is not useful. This flow is characterized in terms of the bulk Richardson number $R_{i}=\left[\left(\rho_{1}\right.\right.$ $\left.\left.-\rho_{2}\right) /\left(\rho_{1}+\rho_{2}\right)\right] g d / \Delta U^{2}$, which will be referred for simplicity Richardson number. The flow is also defined by the dimensionless mean advection that will be useful only in the spatial theory

$$
a=\frac{U_{m}}{\Delta U} .
$$

Considering the stability of two-dimensional parallel flows for three-dimensional disturbances, Yih, ${ }^{25}$ generalized Squire theorem, ${ }^{24}$ without neglecting variations of density or viscosity, which may be continuous or discontinuous, he concluded that the fastest growing mode is two-dimensional. Therefore, we restrict our attention to two-dimensional perturbations of the stream function which are decomposed into normal modes of the form $\phi(y) \exp (i k(x-c t))$, where the eigenfunction $\phi$ is governed by Rayleigh's equation, $k$ denotes the dimensionless wave number and $c$ the phase velocity. In order to ensure that the perturbations decay at infinity $\phi(y)$ is chosen at $y \rightarrow \pm \infty$ to be of the form $\exp ( \pm s k y)(+$ for $y$ $\rightarrow-\infty,-$ for $y \rightarrow+\infty)$, where $s=\operatorname{sgn}\left(k_{r}\right)\left(k_{r}\right.$ is the real part of $k$ ). Imposing the continuity of displacement and pressure at the vorticity and density interfaces give dispersion relation (cf. Drazin and Reid, ${ }^{24}$ or Pouliquen, Chomaz, and Huerre ${ }^{26}$ ) between $k$ and $\omega=k c$, the frequency of the wave

$$
\mathcal{D}\left(k, \omega ; R_{i}, a\right)=(\omega-a k)^{4}+n_{2} k^{2}(\omega-a k)^{2}+n_{0} k^{4}=0,
$$

where

$$
\begin{aligned}
& n_{2}=\frac{-R_{i}}{s k}+\frac{e^{-4 s k}-(2 s k-1)^{2}}{4 k^{2}} \text { and } \\
& n_{0}=\frac{R_{i}}{s k} \frac{\left(e^{-2 s k}+2 s k-1\right)^{2}}{4 k^{2}}, \text { with } s=\operatorname{sgn}\left(k_{r}\right) .
\end{aligned}
$$

\section{TEMPORAL INSTABILITY}

The temporal instability theory considers waves homogeneous in space $(k \in R)$ which develop in time $(\omega \in C, \omega$ $\left.\equiv \omega_{r}+i \omega_{i}\right)$. It correctly describes tilted tank experiments (Thorpe, ${ }^{27}$ Pouliquen, Chomaz, and Huerre ${ }^{26}$ ) where two layers of fluid initially at rest in a horizontal layer are set into relative motion by tilting the tank. We find an expression for the roots of (2) as follows:

$$
\omega=a k \pm\left\{\frac{-n_{2} k^{2} \pm \Delta^{1 / 2}}{2}\right\}^{1 / 2},
$$

with

$$
\Delta=\left(n_{2} k^{2}\right)^{2}-4 n_{0} k^{4} .
$$

The mean advection $a$ [Eq. (1)] in the temporal case acts only as a Doppler shift in frequency, as shown in Eq. (5) and it affects only the real part of $\omega$ in the temporal theory. Therefore, temporal instability will be fully described by considering the intrinsic frequency of the temporal mode, defined as the frequency of the wave seen by an observer moving with the local mean flow $\omega_{r}^{*}=\omega_{r}-a k$ as a function of $k$. Furthermore, since (5) is invariant under the change $\omega(k)=-\bar{\omega}(-\bar{k}) \quad$ (where ${ }^{-}$denotes complex conjugation) without any loss of generality we consider only positive wave numbers. The temporal analysis has already been addressed by Lawrence, Browand, and Redekopp ${ }^{15}$ for a particular broken-line velocity profile in the asymmetric case: 
(a)

$\omega_{i}$

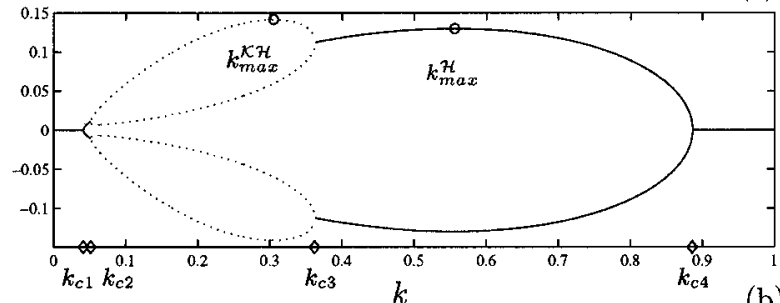

(b)

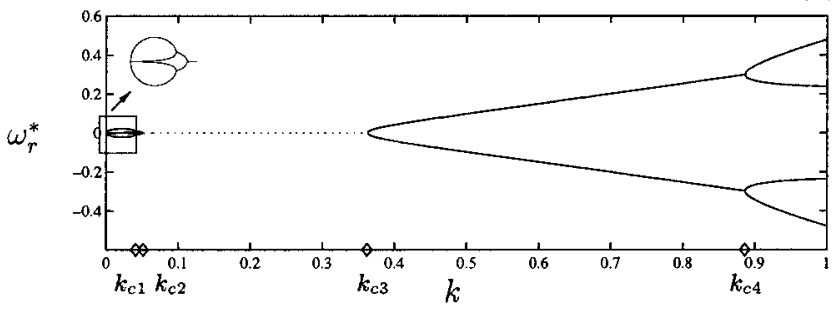

FIG. 3. (a) Temporal growth rate $\omega_{i}$ and (b) intrinsic frequency $\omega_{r}^{*}=\omega_{r}$ $-a k$ with respect to the real wave number $k$ for $R_{i}=0.04$. The dotted curve shows the Kelvin-Helmholtz modes.

the density interface is displaced with respect to the velocity interface and by Smyth and Peltier ${ }^{14}$ for a hyperbolic tangent velocity profile. We have plotted on Fig. 2(a) the stability diagram derived from (2). Without stratification, $R_{i}=0$, there is a unique unstable mode studied by Rayleigh ${ }^{28}$ stationary with respect to the mean flow. When the Richardson number increases, the structure is more complex. To get a better understanding of the unstable modes which exist, we discuss the sign of $\Delta$. When $\Delta>0$ and $n_{2}>0$ then we obtain from (5) two unstable, stationary modes. The most amplified one is the continuation of the mode found by Rayleigh, ${ }^{28}$ the second one is generated by stratification. These instabilities, which we call following Smyth and Peltier ${ }^{14}$ KelvinHelmholtz waves, are stationary with respect to the mean flow and, correspond to the dark gray region on Fig. 2(b). When $\Delta<0$, the unstable modes have intrinsic frequencies with a nonzero real part. Moreover, since under the Boussinesq approximation, the basic flow is invariant under the following reflections, $x \rightarrow-x$ and $y \rightarrow-y$, if $\omega(k)$ is a solution then $\omega(-k)$ is also a solution and as a consequence $-\bar{\omega}(\bar{k})$ is a solution (in the temporal case $k$ is real). Thus when a mode propagating downstream is amplified, a symmetric mode propagating upstream is also unstable with the same growth rate. These propagating unstable modes will be called Holmboe waves. They exist in the light gray region on Fig. 2(b). When the Richardson number increases, two unstable Holmboe regions develop at low and high wave numbers. For $R_{i}=0.07$, the Kelvin-Helmholtz region disappears and the two Holmboe regions merge. For $R_{i}$ larger than 0.07, the Holmboe region moves to larger $k$ but never vanishes [Fig. 2(a)].

For further references, we illustrate the structure of the modes when $R_{i}<0.07$, we consider the case of $R_{i}=0.04$. We plot on Fig. 3 the growth rate $\omega_{i}$ and the intrinsic frequency $\omega_{r}^{*}$ as a function of the wave number $k$. Close to $k=0$, four neutral waves exist, two propagating to the right $\left(\omega^{*}>0\right)$, two to the left $\left(\omega^{*}<0\right)$. When $k=k_{c 1}$, the waves merge two by two and give rise to two unstable Holmboe modes (propa- gating to the right and to the left with $\left.\omega_{i}>0\right)$. When $k$ $=k_{c 2}$, the phase speeds of the two Holmboe modes vanish and two stationary unstable Kelvin-Helmholtz modes appear, the most amplified being the continuation of the homogeneous mode found by Rayleigh, ${ }^{28}$ the less amplified being generated by the stratification [dotted curves on Figs. 3(a) and 3(b)]. At $k=k_{c 3}$, the sequence reverses: the growth rates of the two Kelvin-Helmholtz modes become equal and the character of the instability changes from stationary to oscillatory. These new Holmboe modes have the same growth rate but propagate in opposite directions with respect to the mean flow [Fig. 3(b)]. For $k=k_{c 4}$, the growth rate of the Holmboe modes vanishes and four neutral waves appear. On Fig. 3(a), the temporal growth rate presents two relative maxima $k_{\max }^{\mathcal{K H}}$ and $k_{\max }^{\mathcal{H}}$ associated respectively to KelvinHelmholtz and Holmboe modes. For $R_{i}=0.04$, the KelvinHelmholtz mode is the most unstable (see Fig. 3). On Fig. 2(b), we have plotted the locus of the most unstable wave number as a curve with circles. For $R_{i}^{t}=0.0461$, the most amplified mode switches over from Kelvin-Helmholtz-type to Holmboe type.

\section{ABSOLUTE AND CONVECTIVE INSTABILITIES}

As described in the Introduction in all the laboratory or field situations where the stratified shear flow may be assumed stationary in a particular frame, one should look for the appearance of self-sustained oscillations associated with the absolute nature of the instability in a portion of that flow. ${ }^{19}$ These so-called global modes arise from the building up of energy fluctuation due to the temporal amplification of a wave that does not propagate (of zero group velocity in the frame where the mean flow is stationary). This idea, first developed in plasma physics (Briggs, ${ }^{29}$ Bers $^{30}$ ), is fully discussed in Huerre and Monkewitz ${ }^{19}$ and leads to a discrimination between convective or absolute instability. According to a well established criterion the absolute/convective instability distinction is obtained by studying the behavior of spatial branches $\left(k=k_{r}+i k_{i}\right.$ complex, $\omega$ real $)$, or more generally spatio-temporal branches $\left(k\right.$ and $\omega=\omega_{r}+i \omega_{i}$ complex; $\omega_{r}$ varying and $\omega_{i}$ being constant). The transition occurs when a saddle point of the dispersion relation $\left(k_{0}, \omega_{0}\right)$ crosses the $\omega_{i}$ axis

$$
\begin{aligned}
& \mathcal{D}\left(k_{0}, \omega_{0} ; R_{i}, a\right)=0, \\
& \partial_{k} \mathcal{D}\left(k_{0}, \omega_{0} ; R_{i}, a\right)=0, \\
& \partial_{\omega} \mathcal{D}\left(k_{0}, \omega_{0} ; R_{i}, a\right) \neq 0,
\end{aligned}
$$

with $k_{0}$ the absolute wave number and $\omega_{0}$ the absolute frequency. For shear flow, the dispersion relation contains the non analytic function $\operatorname{sgn}(k)$ [see Eqs. (3)-(5)]. The sign function arises from the constraint that perturbations should decay at $y= \pm \infty$. In order to obtain an analytic function for the dispersion relation (5) in $k$, we restrict the study to $k_{r}$ $>0$ as in Huerre and Monkewitz, ${ }^{31}$ invoking the symmetry $\omega(k)=-\bar{\omega}(-\bar{k})$ and then $s=\operatorname{sgn}\left(k_{r}\right)=1$ in (2)-(4). If the imaginary part of $\omega_{0}, \operatorname{Im}\left(\omega_{0}\right)$, is positive, the flow is absolutely unstable. Conversely if $\operatorname{Im}\left(\omega_{0}\right)$ is negative, the flow is convectively unstable. Conditions (7)-(9) are not explicit 
(a)

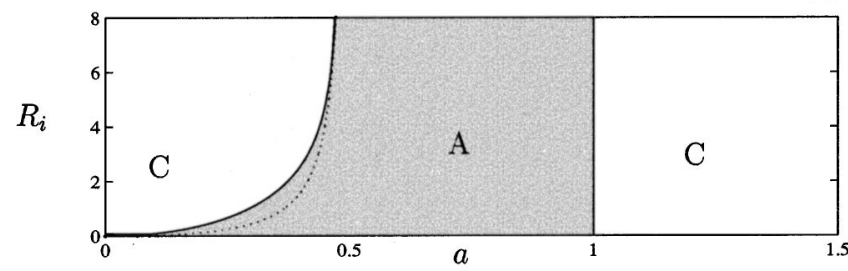

(b)

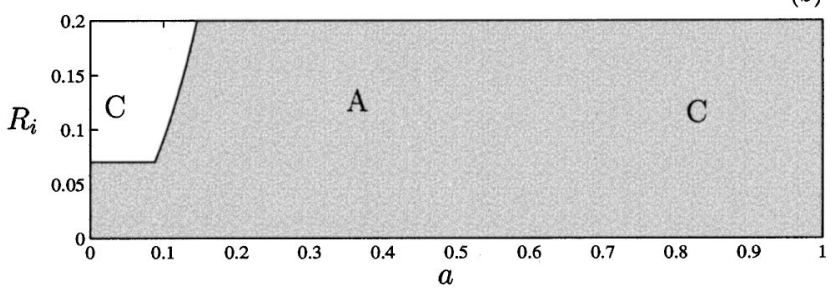

FIG. 4. (a) In light gray, absolute instability, A, in white convective instability, $\mathrm{C}$, in the $\left(a, R_{i}\right)$ plane. The asymptotic behavior calculated in (24) is represented by a dotted curve; (b) zoom of the absolute and convective domains for $R_{i}<0.2$

enough, and the saddle point to be considered must also satisfy a pinching condition of two spatio-temporal branches $k^{ \pm}(\omega)$ arising, respectively from the upper and the lower halves of the $\left(k_{r}, k_{i}\right)$ plane ${ }^{19}$ (i.e., $k_{i}>0$ and $k_{i}<0$ ). In the convective case, the superscript + or - gives the direction of propagation of the wave in the laboratory frame (see Huerre and Monkewitz ${ }^{19}$ for details).

We used the Optimization toolbox of MATLAB to solve the systems $(7)-(9)$. Once $\left(k_{0}, \omega_{0}\right)$ was found, we systematically checked the pinching condition. The domains of absolute and convective instability are plotted on Fig. 4 in the $\left(a, R_{i}\right)$ plane. Due to the symmetry of the problem, when $a$ is changed to $-a, y$ is changed to $-y$, so we focus in the following discussion on $a>0$.

As could be seen on Figs. 4(a) and 4(b), when both streams move in the same direction $(a>1)$, the flow is convectively unstable whatever the value of the Richardson number, i.e., no matter how strong the stratification. When the streams propagate in opposite directions $(a<1)$, two cases must be distinguished. When both Kelvin-Helmholtz and Holmboe modes are unstable, for $R_{i}<0.07$, the flow is absolutely unstable in the whole domain $[0,1[$. Whereas, when only Holmboe modes are unstable, for $R_{i}>0.07$, the flow is convectively unstable for mean advection smaller than a threshold value $a_{c}\left(R_{i}\right)$, and absolutely unstable in a range, $] a_{c}\left(R_{i}\right), 1[$. The structure of Fig. 4, may be easily understood referring to the impulse response of the flow (Fig. 5). Since the impulse response is invariant under Gallilean transformation, its shape is the same whatever the value of the advection parameter. Therefore, a change in the mean advection parameter $a$ corresponds to a change of Gallilean frame. For $R_{i}<0.07$, the absolute-convective transition is associated with the classical picture: a single amplified domain for the impulse response. However, the structure of the wave packet is complicated. It is a result of an hybridization of the Kelvin-Helmholtz and Holmboe modes. The edges of the impulse wave packet move at velocity $a-1$ and $a+1$. For $a$ smaller than unity, the flow is absolutely un-
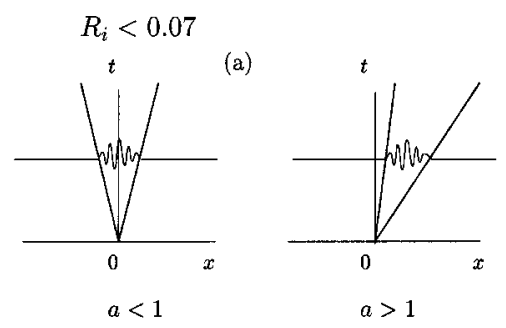

(b)
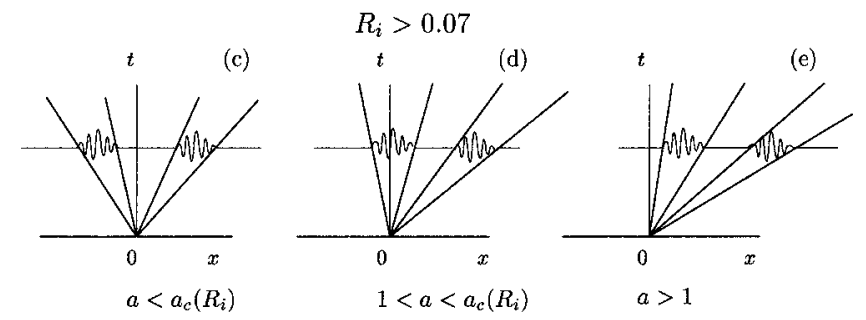

FIG. 5. Sketch of the impulse response in the $(x, t)$ plane. For $R_{i}<0.07$ a single wave packet, (a) absolutely unstable for $a<1$ and (b) convectively unstable for $a>1$. For $R_{i}>0.07$ behavior of the two Holmboe wave packets for $a$ positive varying as indicated on the figure.

stable [Fig. 5(a)]. For $a$ larger than unity the flow is convectively unstable [Fig. 5(b)]. For $R_{i}>0.07$, two Holmboe modes destabilize the flow and the impulse response possesses two amplified regions with edges moving respectively at $a-1$ and $a-a_{c}\left(R_{i}\right)$ and at $a+a_{c}\left(R_{i}\right)$ and $a+1$. The case of the destabilization by two traveling waves was already investigated at the onset of convection in binary fluids. ${ }^{23} \mathrm{In}$ contrast with binary convection which endows the reflectional symmetry $(x \leftrightarrow-x)$ (see Huerre et al. ${ }^{19}$ Kolodner, Surko, and Williams ${ }^{23}$ ), the free mean advection parameter, $a$, of the present model flow, breaks the reflectional symmetry and makes the discussion richer. Three possible configurations may be encountered depending on the value of $a$ positive (the case $a<0$ being symmetric). For $a<a_{c}\left(R_{i}\right)$ [Fig. 5(c)] the wave packets move away from the source to the left and the other to the right and the flow is, therefore, convectively unstable [white region on the left of the Fig. 4(a)]. When $a_{c}\left(R_{i}\right)<a<1$ [light gray region in Fig. 4(a)] the left moving wave packet is now making the flow absolutely unstable since it is exponentially growing at the impulse location [Fig. 5(d)]. The absolute instability is, therefore, triggered by the Holmboe wave associated with the lower layer. When $a>1$, both waves packets propagate to the right [Fig. $5(\mathrm{e})$ ], and the flow is again convectively unstable [right white region in Fig. 4(a)].

Two characteristics in the flow behavior visible on Fig. 4 should be pointed out. First, $a=1$ defines a transition from absolute to convective instability for all $R_{i}$. For $R_{i}=0$, this result was obtained by Balsa ${ }^{32}$ and by Bechert. ${ }^{33}$ In Sec. VI, a large $k$ asymptotic expansion will explain the particular significance of the $a=1$ value. Second, the transition at $R_{i}$ $=0.07$ from a single wave packet to a double wave packet is abrupt as the Kelvin-Helmholtz modes become stable.

\section{SPATIAL INSTABILITY}

Spatial waves have been much debated due to their unbounded behavior at $|x| \rightarrow \infty$ (Drazin and Reid, ${ }^{24}$ pp. 147- 
(a)

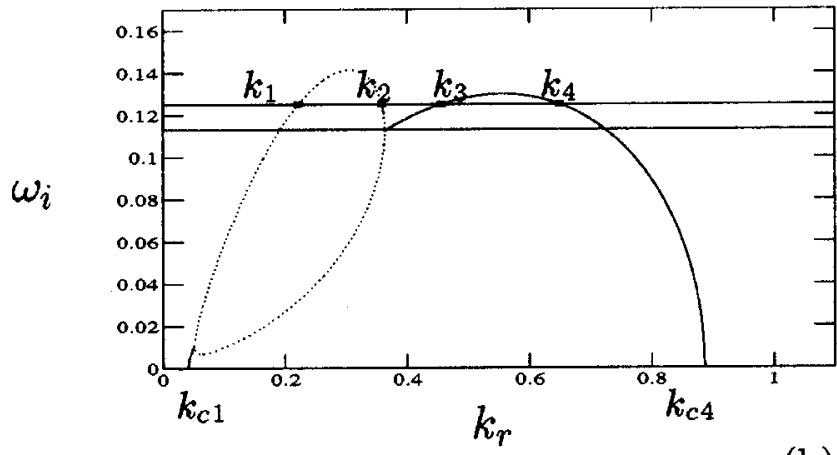

(b)

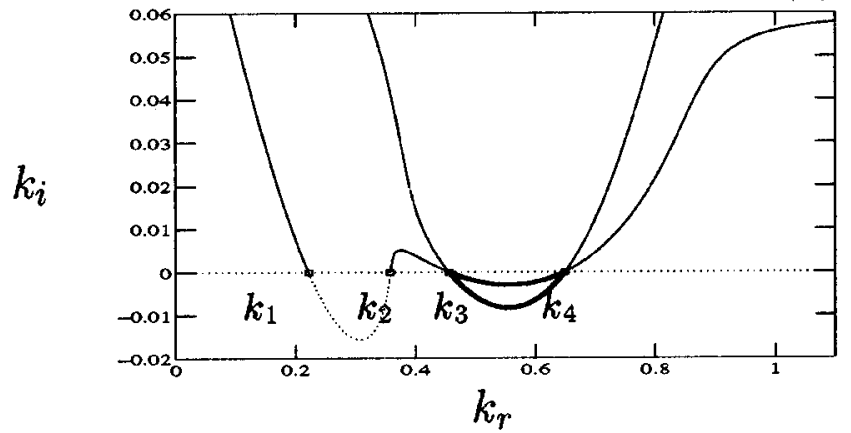

(c)

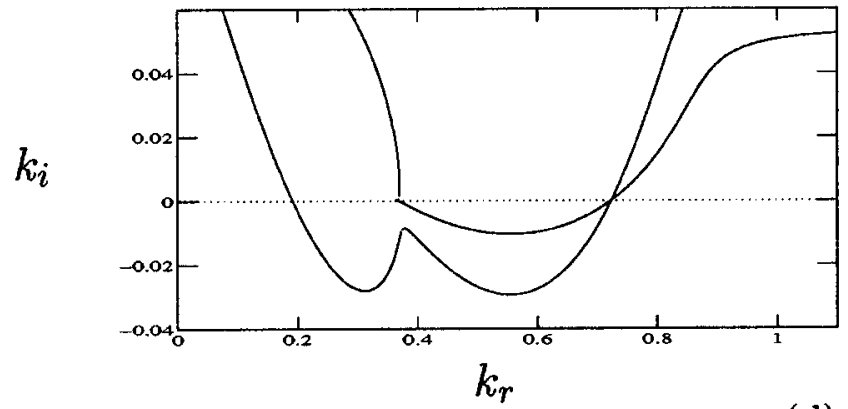

(d)

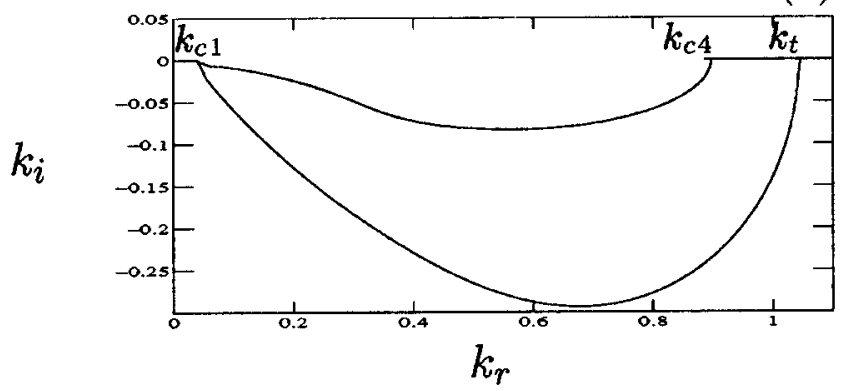

153). However when the flow is convectively unstable, they appropriately describe the asymptotic response as $t \rightarrow \infty$ to a spatially localized harmonic source turned on at the origin of time (see $\mathrm{Chomaz}^{34}$ ). In this case, initial transients, due to the switching on of the harmonic forcing, are advected away from the forcing excitation. The spatially amplified waves radiated from the source are left behind this transient wave packet and may be interpreted in terms of spatial causality. In absolutely unstable flows, transients exponentially growing in time do not propagate away and overwhelm the forcing response. As for temporal theory, the structure of spatial instability branches will differ for $R_{i}$ larger or smaller than $\omega_{i}=0.125$

$\omega_{i}=0.113$

$\omega_{i}=0.125$

$\omega_{i}=0.113$

$\omega_{i}=0$
FIG. 6. In dotted curve, Kelvin-Helmholtz branches. In solid curve Holmboe branches (a) temporal growth rate $\omega_{i}$ function of the wave number $k_{r}$ for $R_{i}=0.04$; spatiotemporal branches for $R_{i}=0.04$ and $a=1.1 \omega_{r}$ varies between 0 and 1.5 by small increments (b) for $\omega_{i}$ $=0.125$, (c) pinching of amplified branches and exchange of branches for $\omega_{i}=0.113$, and (d) hybrid spatial branches $\omega_{i}=0$.
0.07 . The case $R_{i}<0.07$ will be more complex since KelvinHelmholtz and Holmboe modes that are well separated in the temporal theory will mix and following Pawlak and Armi, ${ }^{22}$ we will name the domain $R_{i}<0.07$, the hybrid region.

\section{A. Hybrid region}

For $R_{i}<0.07$, and in the particular case of wedge flows $(a=1)$, Pawlak and $\mathrm{Armi}^{22}$ found numerically, using the Taylor-Goldstein equation, two propagating spatially growing modes. They called them hybrid modes since in the spatial case no straightforward criteria discriminates between 
Kelvin-Helmholtz and Holmboe modes. In order to gain some insight into these hybrid modes, and to illustrate the existence of saddle points in the dispersion relation that do not lead to an absolute-convective transition, we consider the deformations of the spatio-temporal branches as the $\omega$ contour is lowered from high values of $\omega_{i}$ toward $\omega_{i}=0$, in the case $R_{i}<0.07$ and $a>1$. We plot on Figs. 6(b) $-6(\mathrm{~d})$, only the downstream amplified branches. All the branches are obtained by solving numerically the dispersion relation (2) using MATLAB Optimization routines for a particular set of fixed parameters $\omega_{i}, R_{i}$, and $a$ and with $\omega_{r}$ varying. These branches are parametrized by $\omega_{r}$ which varies by small increments (see Loiseleux, Chomaz, and Huerre for details ${ }^{35}$ ). Figure 6(a) reproduces Fig. 3(a) and presents the temporal growth rate for $R_{i}=0.04$ and $a=1.1$. When $\omega_{i}=0.125$, the wave numbers $k_{1}, k_{2}, k_{3}$, and $k_{4}$ belonging to the unstable temporal branches [Fig. 6(a)], also belong to the downstream spatio-temporal branches for $\omega_{i}=0.125$ plotted on Fig. 6(b). We note two downstream amplified branches on Fig. 6(b). For the first branch, the two unstable parts in the dotted curve between $k_{1}$ and $k_{2}$ and in the heavy curve between $k_{3}$ and $k_{4}$, may be associated respectively to Kelvin-Helmholtz and Holmboe modes. The second downstream unstable branch is unstable between $k_{3}$ and $k_{4}$, which corresponds to the most spatially amplified Holmboe branch. The least amplified Kelvin-Helmholtz branch is associated with a damped spatio-temporal branch (i.e., $\left.k_{i}>0\right)$ since $\omega_{i}$ $=0.125$ is larger than its maximum growth rate $\left(\omega_{i}\right.$ $\sim 0.113$ ). While lowering the $\omega_{i}$ contours, we may follow the deformations of those branches. In this process, a saddle point is encountered for $\omega_{i}$ slightly larger than 0.113 , but it does not involve an interaction between two branches arising from different part of the $k_{i}$ plane. This saddle point does not contribute to the impulse response. ${ }^{19}$ The branches plotted on Fig. 6(c) are a result of this interaction. The effect of this saddle point has been to "hybridize" the two KelvinHelmholtz modes with the two Holmboe modes, but all four modes keep propagating information downstream. Finally, on Fig. 6(d), we obtain the two downstream amplified spatial branches ( $\omega$ real). Another saddle point which is once again not a pinch point is encountered for $\omega_{i} \sim 0.0099$ when the small wave number Holmboe modes destabilize $[k$ $\in\left[k_{c 1}, k_{c 2}\right]$ Fig. 3(b)]. This continuous switching of branches justifies the terminology hybrid mode.

The structure of the hybrid branch near $k=k_{t}$ plotted on Fig. 6(d) is surprising in the sense that a neutral mode (i.e., $\omega$ and $k$ real) is a spatio-temporal wave that belongs both to the temporal and to the spatial branches as is the case for $k$ $=k_{c 1}$ and $k=k_{c 4}$. Therefore, it should appear both as a crossing of the temporal branch with the $\omega_{i}=0$ axis [Fig. 6(a) ] and as a crossing of the spatial branch with the $k_{i}=0$ axis [Fig. 6(d)]. But the most amplified branch seems to cross at $k=k_{t}$ and to have no temporal counterpart. When $\omega_{i}$ is small but nonzero the spatio-temporal branch crosses the $k_{i}=0$ axis close to $k=k_{c 4}$ as it should, but prior to this crossing it makes a large turn about close to $k_{t}$ and then reaches $k_{c 4}$ parallel to the $k_{i}=0$ axis [continuous curve on Fig. 7(a)]. The smaller $\omega_{i}$, the closer this final portion of the spatio-temporal branch to the $k_{i}$ axis. Finally when $\omega_{i}$ van-
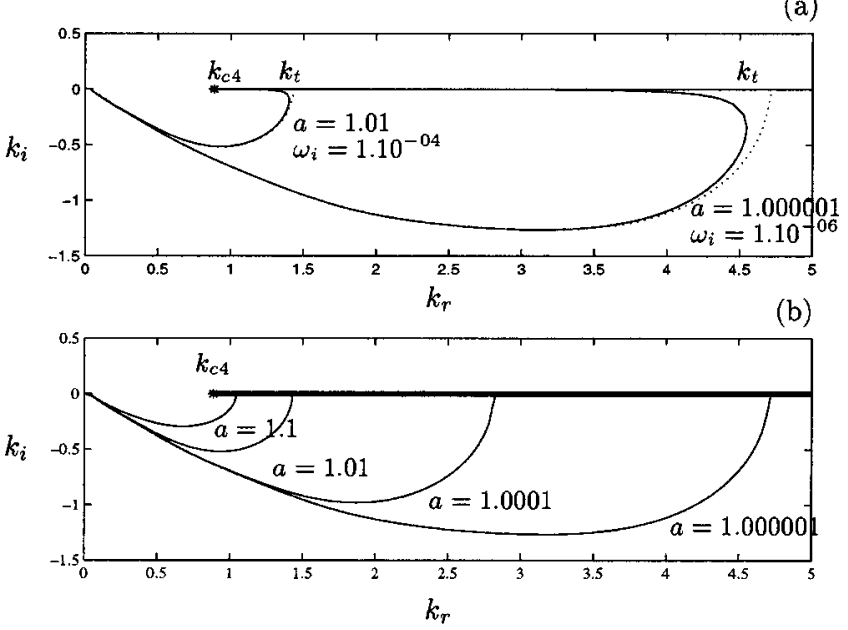

FIG. 7. (a) Comparison between the most amplified spatio-temporal branches in solid curve at small values of $\omega_{i}$ and between the spatial branches $\omega_{i}=0$ in dotted curve for two values of $a, a=1.01$ and $a$ $=1.000$ 001. (b) Behavior of the most amplified hybrid spatial branch, Fig. 6(d), for $R_{i}=0.04$ and for $a=1.1, a=1.01$ and $a=1.0001$, and $a$ $=1.000001 ; k_{c 4}$ is the neutral wave number defined on Fig. 3 .

ishes, the branch becomes singular [dotted curve on Fig. 7(a)] and seems to cross at $k=k_{t}$. Clearly, the limit $\omega_{i}=0$ is singular since the most unstable spatial branch collapses on the $k_{i}=0$ axis at $k=k_{t}$ [Fig. 7(a)]. Therefore, $k=k_{t}$ is only a pseudo-intersection of the most unstable branch with the $k_{i}$ $=0$ axis since $k=k_{t}$ is never reached. The dispersion relation (5) exhibits a square root behavior at the neutral wavenumber, $k_{c 4}$ (see also Fig. 3). Therefore, a branch cut lies from $k_{c 4}$ to infinity (Fig. 7) in the $k$ plane. This singular behavior is reponsible for the $a=1$ transition. When $a$ decreases from $a=1.1$ to $a=1.000001$ [Fig. 7(b)], the pseudo-intersection, $k_{t}$, of the hybrid branch with the branch cut moves to large value. At $a=1, k_{t}$ tends to infinity, and the pinching of the real axis occurs at $+\infty$ and not as usual at a finite saddle point. In fact, an infinity of spatial branches, shown on Fig.

(a)

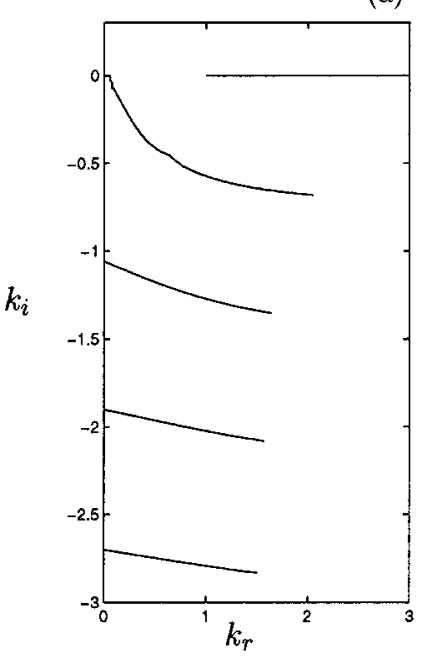

(b)

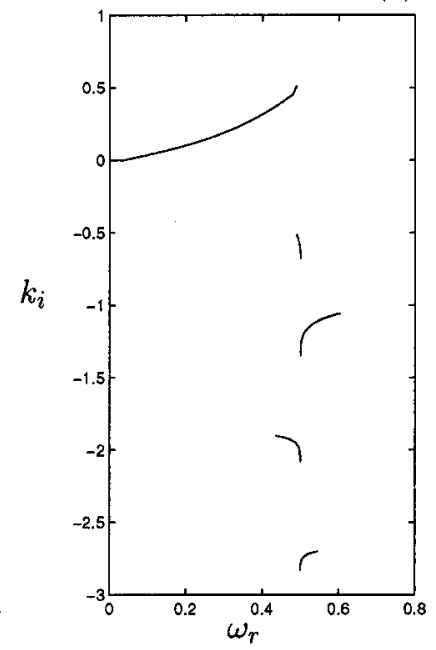

FIG. 8. (a) Simultanously pinching of several branches at $a=1$ and $R_{i}$ $=0$. (b) Bechert Christmas tree (Ref. 33) for $a=1$ and $R_{i}=0$. 
(a)
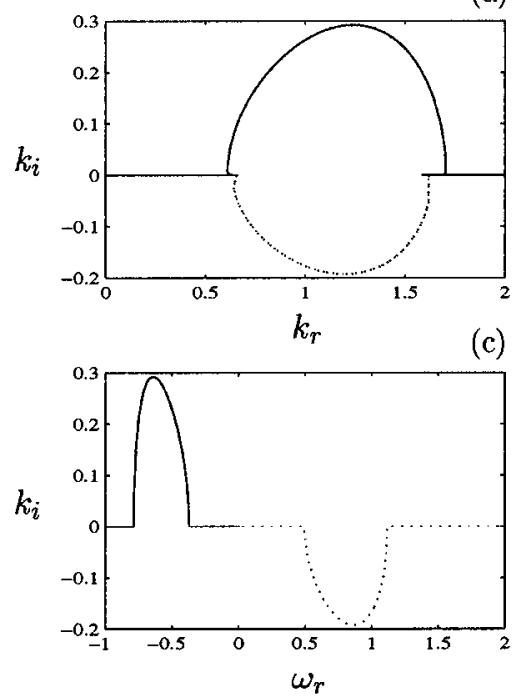

(b)

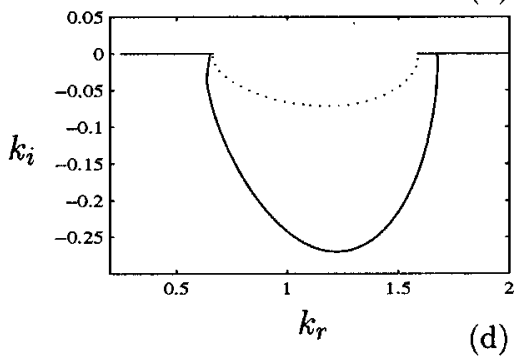

(d)

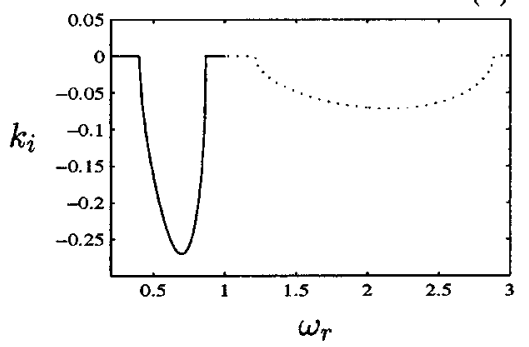

FIG. 9. Amplified spatial Holmboe branches for $R_{i}$ $=0.5$. In solid curve Holmboe branch associated with the lower frequency, in dotted curve Holmboe mode associated with the greater frequency; (a) $k_{i}$ function of the wave number $k_{r}$ for $a=0.1$ and (b) for $a=1.2$; (c) $k_{i}$ function of the frequency $\omega_{r}$ for $a=0.1$, (d) for $a$ $=1.2$. 8(a), go through a similar transition at the same time [Fig. 8(a)]. Plotted in the $\left(k_{i}, \omega_{r}\right)$ plane for the particular case $R_{i}$ $=0$, these branches evoke an upside down Christmas tree in Bechert's imagination $^{33}$ [Fig. 8(b)]. This singularity leads to an essential singularity of the dispersion relation. In Sec. VI, we will see that this behavior persists for all values of the Richardson number.

\section{B. Holmboe spatial modes}

When $R_{i}>0.07$, the Kelvin-Helmholtz mode is stabilized and two counterpropagating Holmboe waves destabilize the flow. As a result, the instability is convective in two distinct domains, $0<a<a_{c}\left(R_{i}\right)$ and $a>1$ as shown on Fig. 4. The structure of the spatial branches belonging to each of the convectively unstable domains are shown on Fig. 9.

When the mean advection $a$ is smaller than $a_{c}\left(R_{i}\right)$, the response to an impulse consists of two wave packets traveling in opposite directions [Fig. 5(c)]. The mode traveling with the negative group velocity is amplified upstream and the corresponding amplified spatial branch is characterized by $k_{i}>0$. It is represented on Fig. 9(a) by a solid curve. In contrast the other Holmboe mode travels downstream and the corresponding amplified spatial branch represented by a dotted curve on Fig. 9(a) is such that $k_{i}<0$. On Fig. 9(c) the spatial growth rates are represented as a function of the frequency. Waves moving upstream are represented by negative $\omega_{r}$ values since $k_{r}$ is assumed positive by convention.

When the mean advection $a$ is larger than unity, the flow is convectively unstable and the impulse response is associated with two wave packets, both traveling downstream [Fig. $5(\mathrm{e})]$. The mode traveling with the smaller group velocity is the most spatially amplified, and is represented by a solid curve on Figs. 9(b) and 9(d). This contrasts with the temporal theory for which both Holmboe modes have the same amplification rates. Qualitatively, these results may be understood using the Gaster transformation, ${ }^{36}$ which gives, in the limit of small amplification rates, the link between the spatial and temporal amplification rates at the same real wave number $k_{r}$

$$
\frac{\omega_{i}}{k_{i}} \sim \frac{\partial \omega_{r}}{\partial k_{r}}\left(k_{r}\right) \sim-v_{g} .
$$

However, as discussed in a later section, much care must be taken using Eq. (10) since the Gaster transformation is valid only under certain assumptions. ${ }^{36}$ Figure 9 (d) presents the spatial growth rate as a function of the wave frequency $\omega_{r}$. Since both waves are propagating downstream, $\omega_{r}$ is positive, but the range of frequencies associated with the slower moving wave is smaller. For the slower moving wave (higher growth rate and smaller frequency), the eigenfunction is more intense in the slower moving layer [Fig. 10(b)]. Conversely, the other spatially unstable branch, with a smaller spatial growth rate and a larger frequency is localized in the

(a)
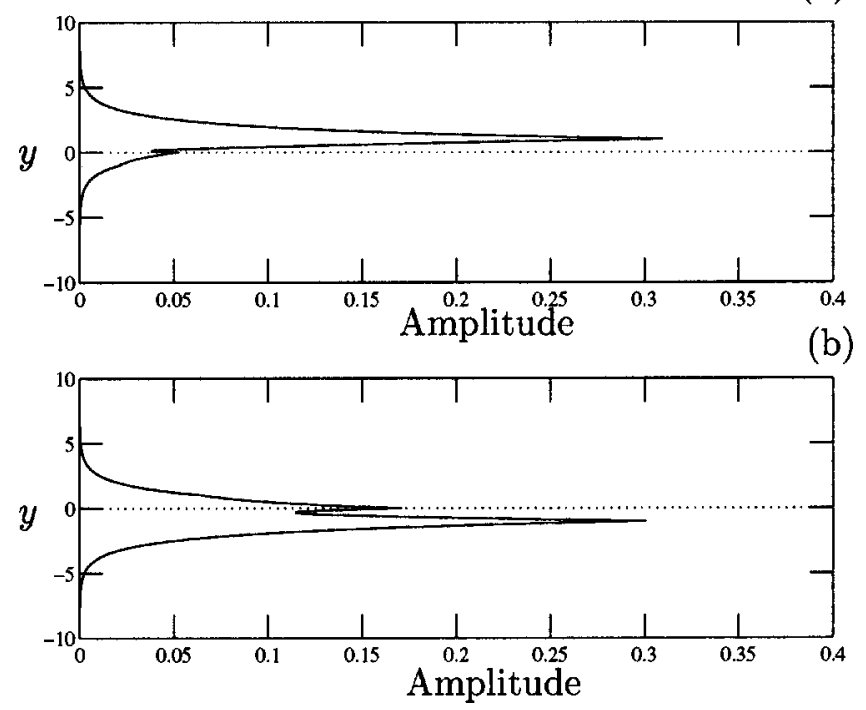

FIG. 10. Amplitude of the eigenfunction for $R_{i}=0.5, a=1.2$ associated with each most amplified wave number on each Holmboe branch [see Fig. 9(b)]. On (a), eigenfunction associated with the Holmboe mode traveling with the faster layer (i.e., the upper layer here) (b) eigenfunction of the most amplified wave number traveling with the lower layer. 
fast moving stream [Fig. 10(a)]. The difference in spatial growth rates suggests that the Holmboe mode concentrated in the slower layer [Fig. 10(b)] may dominate in experiments when broadband forcing is applied at the inlet. However, since Holmboe modes are unstable over different frequency bands as shown on Figs. 9(c) and 9(d), an appropriate forcing at the inlet may select experimentally one or the other mode.

\section{ASYMPTOTIC RESULTS}

In the inviscid approximation, the configuration of Fig. 1 is unstable to wavelength that goes to zero $(k \rightarrow \infty)$ when the strength of the stratification increases, i.e., when $R_{i} \rightarrow \infty$ [Fig. 2(a)]. In this section, we carry out an asymptotic expansion of the dispersion relation in the limit $k_{r} \rightarrow \infty$. We will consider first the case $R_{i} \rightarrow \infty$ and in a second part $R_{i}$ finite. This analysis explains on physical grounds the convectiveabsolute transitions computed numerically.

Neglecting all terms of order less than $\exp \left(-3 k_{r}\right)$, the complex frequency (5) becomes with $k$ real or complex but $k_{r}$ large

$$
\omega \simeq a k \pm \frac{k}{\sqrt{2}} \sqrt{\frac{R_{i}}{k}+\frac{(2 k-1)^{2}}{4 k^{2}} \pm \sqrt{\Delta_{\mathrm{asymp}}}}+o\left(e^{-3 k_{r}}\right) .
$$

In the above expression, $\Delta_{\text {asymp }}$ is the asymptotic value of (6) defined by

$$
\begin{aligned}
\Delta_{\text {asymp }} \simeq & \left(\frac{R_{i}}{k}-\frac{(2 k-1)^{2}}{4 k^{2}}\right)^{2} \\
& -\frac{2 R_{i}(2 k-1) k e^{-2 k}}{k^{4}}+o\left(e^{-3 k_{r}}\right) .
\end{aligned}
$$

\section{A. Asymptotics at large $\boldsymbol{R}_{\boldsymbol{i}}$}

Temporally amplified modes ( $k$ real) are obtained when $\Delta_{\text {asymp }}(12)$ is negative. For large $k$ away from the unstable band of wave numbers, $\Delta_{\text {asymp }}$ is $\mathcal{O}\left(1 / k^{4}\right)$. However, for the particular wave number $k_{m}$ such that

$$
R_{i}=\frac{\left(2 k_{m}-1\right)^{2}}{4 k_{m}}
$$

the function $\Delta_{\text {asymp }}$ is of order $e^{-2 k_{m}} / k_{m}$. For this particular value of $k_{m}$, we obtain $\Delta_{\text {asymp }}$ minimal and negative. The expression (13) has been already derived by Caulfield ${ }^{37}$ in the form $R_{i}=k_{m}-1$. The most amplified wave number $k_{m}$ is represented by a dotted curve on Fig. 2(a). Moreover, as can be seen on Fig. 2(a), the stronger the stratification, the narrower the band of temporally unstable wave numbers. Therefore, in order to obtain the asymptotic expansion of the dispersion relation, we let $R_{i}$ goes to infinity and we seek the wave numbers close to $k_{m}$ for which, the two terms of righthand side of (12) have the same order and $\Delta_{\text {asymp }}$ negative. Therefore, we write

$$
k=k_{m}+\epsilon k^{\prime},
$$

with $k_{m}$ given by (13) $k^{\prime}$ real or complex and the gauge $\epsilon$ determined by the dominant balance

$$
\epsilon=\sqrt{k_{m}} e^{-k_{m}}
$$

The dispersion relation (11) may be rewritten at order $\epsilon$ as followed with $s_{1}= \pm 1$ and $s_{2}= \pm 1$ independent

$$
\begin{aligned}
\omega= & a k_{m}+\epsilon a k^{\prime}+s_{1} k_{m}-\frac{s_{1}}{2}+\epsilon s_{1} k^{\prime}\left(\frac{3}{4}-\frac{1}{8 k_{m}}\right) \\
& +\epsilon s_{2} \frac{2 k_{m}+1}{8 k_{m}} \sqrt{k^{\prime 2}-\frac{8 k_{m}\left(2 k_{m}-1\right)}{\left(2 k_{m}+1\right)^{2}}} .
\end{aligned}
$$

The unstable temporal wave numbers $\left(k^{\prime}\right.$ real $)$ are obtained for $k^{\prime 2}-8 k_{m}\left(2 k_{m}-1\right) /\left(2 k_{m}+1\right)^{2}$ negative, which specifies the band of unstable wave numbers

$$
k=k_{m}+\epsilon k^{\prime}
$$

with

$$
k^{\prime} \in\left[-\frac{\sqrt{8 k_{m}\left(2 k_{m}+1\right)}}{2 k_{m}+1}, \frac{\sqrt{8 k_{m}\left(2 k_{m}+1\right)}}{2 k_{m}+1}\right],
$$

then at leading order in $\epsilon$ the frequency of an unstable wave number $k(17)$ is given by

$$
\omega_{r}=a k_{m}+\epsilon a k^{\prime}+s_{1} k_{m}-\frac{s_{1}}{2}+\epsilon s_{1} k^{\prime}\left(\frac{3}{4}-\frac{1}{8 k_{m}}\right),
$$

with a growth rate

$$
\omega_{i}=\epsilon \frac{2 k_{m}+1}{8 k_{m}} \sqrt{\frac{8 k_{m}\left(2 k_{m}-1\right)}{\left(2 k_{m}+1\right)^{2}}-k^{\prime 2}} .
$$

The complex frequencies $\left(\omega_{r}+i \omega_{i}\right)$ of the temporally amplified Holmboe modes associated with the most unstable wave number $k_{m}$ are obtained letting $k^{\prime}=0$ in (18) and (19)

$$
\omega_{m}^{ \pm}=\left\{a k_{m} \pm\left(k_{m}-\frac{1}{2}\right)\right\}+i \epsilon \frac{\sqrt{2 k_{m}-1}}{\sqrt{8 k_{m}}},
$$

the associated group velocity is obtained calculating $\partial \omega / \partial k$ for $\omega$ given by (16) and evaluated at $k^{\prime}=0$

$$
\begin{aligned}
v_{G}\left(k_{m}\right)=\frac{\partial \omega}{\partial k}\left(k_{m}\right) & =\frac{1}{\epsilon} \frac{\partial \omega}{\partial k^{\prime}}(0) \\
& =a \pm\left(\frac{3}{4}-\frac{1}{8 k_{m}}\right) \rightarrow a \pm \frac{3}{4} .
\end{aligned}
$$

The results (17)-(21) match quantitatively well with numerical calculations.

The absolute unstable branch may be computed using the dispersion relation (16) derived for $k^{\prime}$ real or complex. Imposing $\partial \omega / \partial k=0$ with $\omega$ and $k=k_{m}+\epsilon k^{\prime}$ linked by (16), give the absolute frequency $\omega_{0}$ and wave number $k_{0}^{\prime}$

$$
k_{0}^{\prime}=\frac{\left(a+s_{1}\left(\frac{3}{4}-\frac{1}{8 k_{m}}\right)\right) \frac{\sqrt{8 k_{m}\left(2 k_{m}-1\right)}}{\left(2 k_{m}+1\right)}}{\sqrt{\left(a+s_{1}\right)\left(a+s_{1}\left(\frac{1}{2}+\frac{1}{4 k_{m}}\right)\right)}},
$$



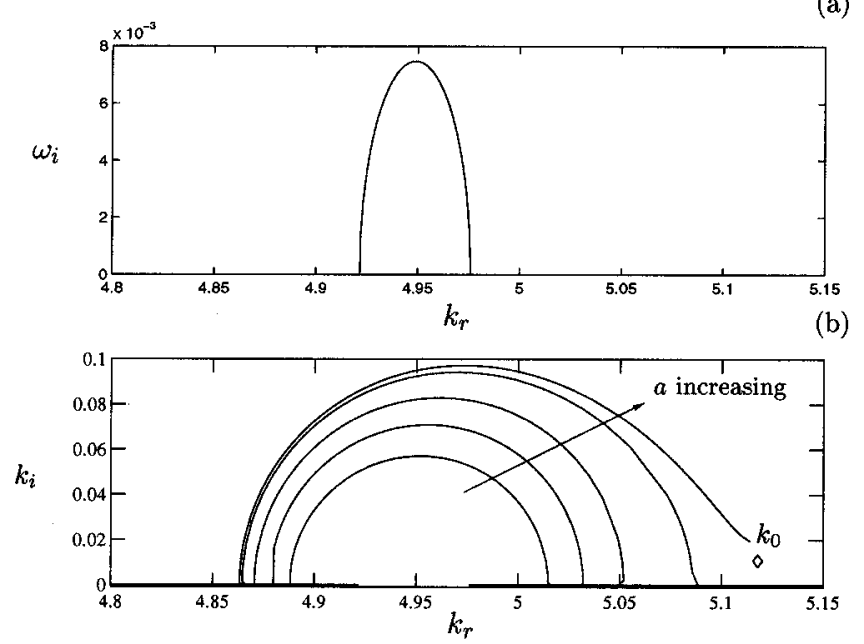

FIG. 11. Comparison between (a) the temporal amplification rate $\omega_{i}$ and (b) the spatial amplification rate $k_{i}$ for the Holmboe branch traveling with the lower layer for $R_{i}=4$, and several values of the advection parameter $a$ $=0.42, a=0.43, a=0.435$, and $a=0.438$ where the pinching $k_{0}$ occurs.

$$
\begin{aligned}
\omega_{0}= & a k_{m}-s_{1}\left(k_{m}-\frac{1}{2}\right)+\epsilon k_{0}^{\prime} \\
& \times\left[\frac{\left(a+s_{1}\right)\left(a+s_{1}\left(\frac{1}{2}-\frac{1}{4 k_{m}}\right)\right)}{a+s_{1}\left(\frac{3}{4}-\frac{1}{8 k_{m}}\right)}\right] .
\end{aligned}
$$

The absolute-convective transition corresponds to the imaginary part of $\omega_{0}$ equals to zero, which yields according to (13) and with $s_{1}= \pm 1$

$$
\begin{aligned}
a_{c}\left(R_{i}\right) & = \pm\left(\frac{1}{2}-\frac{1}{4 k_{m}}\right) \\
& = \pm\left(\frac{1}{2}-\frac{2}{1+R_{i}+\sqrt{\left(1+R_{i}\right)^{2}-1}}\right)
\end{aligned}
$$

and

$$
a= \pm 1 .
$$

The transition $a=1$ (25) obtained at large $R_{i}$, corresponds exactly with the numerically computed threshold [Fig. (4)]. The second transition $a_{c}\left(R_{i}\right)$ given on Eq. (24) is represented by a dotted line on Fig. 4(a), the asymptotic calculus matches remarkably well the numerical calculation. At the limit $k_{m}$ $\rightarrow \infty, a_{c}$ tends to $1 / 2$.

Moreover, when the advection parameter $a$ approaches one of the two thresholds (24) or (25), $\left|k_{0}^{\prime}\right|$ tends to infinity, this suggests that the spatial growth rate is greater than the temporal one near the threshold. Numerically, we compare near the first threshold $a_{c}\left(R_{i}\right)$ on Fig. (11) the spatial and temporal amplification rates of the Holmboe mode amplified upstream $\left(k_{i}<0\right)$, for $R_{i}=4$. We note that the band of temporal unstable wave numbers, which matches exactly with (17), is smaller than in the spatial case for the advection parameter approaching the transition $a_{c}=0.4368$. The differ- ence of growth rates in temporal and spatial case near the thresholds enhances the failure of the Gaster transformation [Eq. (10)] in those cases (see Sec. VII).

\section{B. Asymptotics at finite $\boldsymbol{R}_{\boldsymbol{i}}$}

The dispersion relation displays two amplified and two damped solutions. The branch leading to the pinching is the Holmboe mode traveling with the smaller phase velocity [Fig. 5(d)]. Choosing the principal square root such that $Z^{1 / 2}$ maps the complex $Z$-plane cut along the negative real axis onto the half space $R_{e}\left(Z^{1 / 2}\right)>0$, the amplified spatial branch downstream is recovered solving

$$
\begin{aligned}
\omega \simeq & a k-\frac{k}{\sqrt{2}} \sqrt{\frac{R_{i}}{k}+\frac{(2 k-1)^{2}}{4 k^{2}}+\sqrt{\Delta_{\text {asymp }}}} \\
& +o\left(e^{-3 k_{r}}\right) .
\end{aligned}
$$

The threshold $a=1$ (25) is independent on $R_{i}$. Moreover as shown in the hybrid region in Fig. 7, when the advection parameter $a$ tends to unity, the absolute wave number $k_{0}$ pinches at infinity for any value of $R_{i}$. We propose to capture this behavior assuming $R_{i}$ of order unity and $k$ complex but $k_{r}$ large. Under this assumption, the square term in $\Delta_{\text {asymp }}$ given in (12) is always dominant with respect to the second term. Neglecting the terms of order equal or less than $e^{-2 k} / k^{2}$, we obtain

$$
\Delta_{\text {asymp }} \sim\left(\frac{R_{i}}{k}-\frac{(2 k-1)^{2}}{4 k^{2}}\right)^{2}+O\left(\frac{e^{-2 k}}{k}\right) .
$$

For $k$ complex but $k_{r}$ large the dispersion relation becomes

$$
\omega=a k-\frac{2 k-1}{2} .
$$

The absolute frequency and wave number are obtained solving $\partial \omega / \partial k=0$

$$
\frac{\partial \omega}{\partial k}=a-1=0 .
$$

For $a=1$, the absolute wave number tends to $\omega_{0}=1 / 2$ and the absolute wave number $k_{0}$ is not determined. The value of this absolute frequency is retrieved numerically, in this paper we have only represented the classical Bechert Chritmas tree, ${ }^{33}$ (Fig. 8) (that means for $R_{i}=0$ ) included in this analysis.

As a conclusion of the asymptotic studies, the absolutely unstable domain goes to $a=1 / 2$ to $a=1$ as $R_{i}$ goes to infinity. At the trailing edge the first threshold (24) tends to $1 / 2$, the group velocity of a pure gravity wave with a phase velocity equals to 1 . Indeed the computed eigenfunction corresponding to this mode [given in Fig. 12(b)] has a large amplitude only around the density interface and indeed, corresponds to a gravity wave marginally destabilized by the interaction with the density interface. At $a=3 / 4$, the most amplified modes differ from gravity waves, the associated eigenmode being intense both at $y=1$ and $y=0$ [Fig. 12(a)]. At the leading edge of the wave packet, $a=1$, only the layer 
(a)

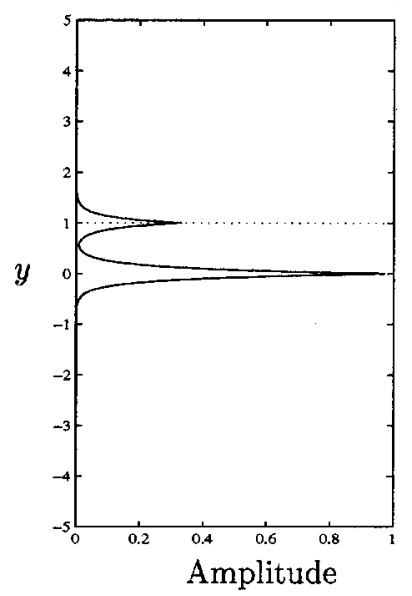

(b)

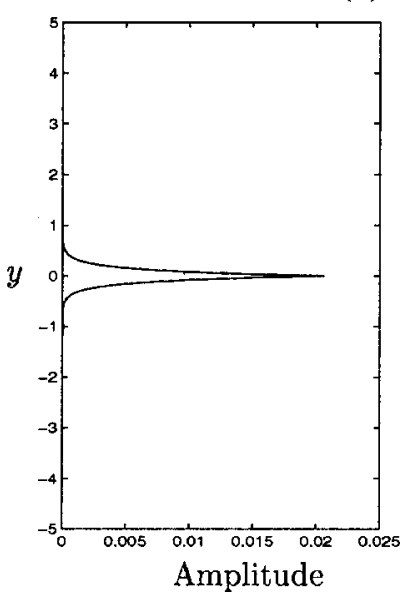

FIG. 12. (a) Amplitude of the eigenfunction associated with $\omega_{m}^{+}$(20) and with the most amplified wave number $k_{m}$ for $R_{i}=8$. (b) Amplitude of the eigenfunction associated with the wave number $k_{0}$ and with the absolute frequency $\omega_{0}$ [see on Fig. 12(b)] for $R_{i}=4$ and $a=0.438$, that means for the first convective-absolute transition.

at $y=-1$ is active, and the mode is insensitive to $R_{i}$, but since the pinching occurs at infinity no eigenmode may be exhibited.

\section{DISCUSSION AND CONCLUSION}

For $R_{i}=4$ and for different values of the advection parameter $a$ from 0.2 to 1.5 , we have plotted on Fig. 13 (resp. Fig. 14), the spatial amplification rates $k_{i}$ of the right-going Holmboe mode (resp. left-going mode) as a function of the intrinsic frequency $\omega_{r}^{*}$ obtained solving numerically the dispersion relation (2). The right-going and the left-going Holmboe modes are represented by a solid curve on Figs. 13 and 14 . We will compare those branches with predictions of

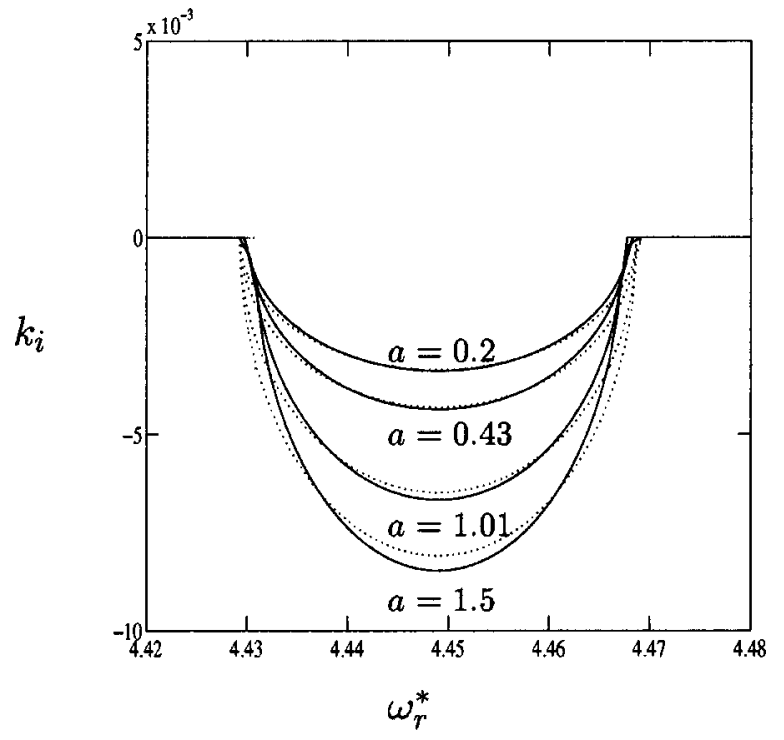

FIG. 13. Spatial growth rate of the right-going Holmboe wave versus the intrinsic frequency $\omega_{r}^{*}$, for $R_{i}=4$ and different values of the advection parameter $a, a=1.5, a=1.01, a=0.43$, and $a=0.2$. In solid curve, Holmboe branches obtained by solving the dispersion relation, in dotted curve by following the Gaster transformation (10).

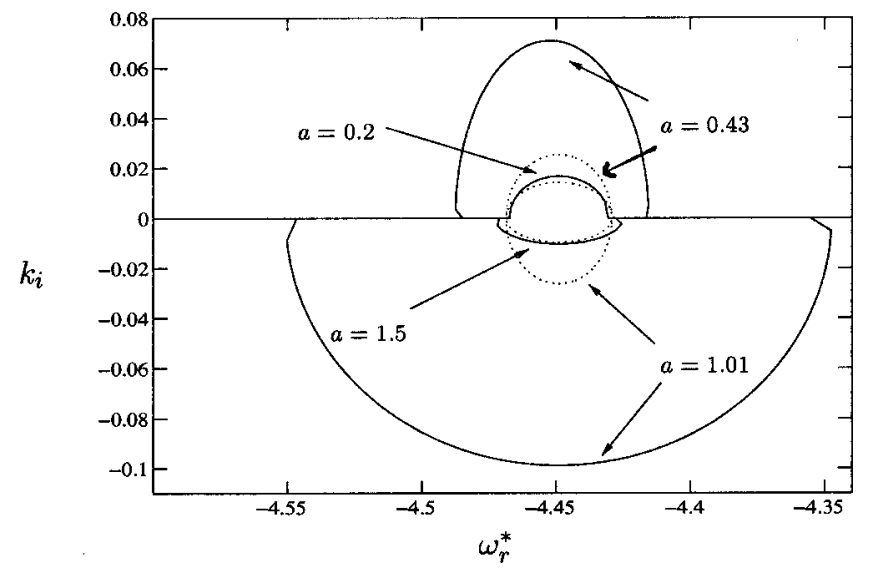

FIG. 14. Spatial growth rate for the left-going Holmboe wave versus the intrinsic frequency $\omega_{r}^{*}$, for $R_{i}=4$ and different values of the advection parameter $a, a=1.5, a=1.01, a=0.43$, and $a=0.2$. In solid curve, Holmboe branches obtained by solving the dispersion relation, in dotted curve by following the Gaster transformation (10).

the spatial amplification rates obtained transforming the temporal growth rate using Gaster transformation ${ }^{36}$ recalled here. For a given real wave number $\tilde{k}_{r}$, the temporal analysis leads to $\omega=\omega_{r}\left(\widetilde{k}_{r}\right)+i \omega_{i}\left(\widetilde{k}_{r}\right)$ complex solution of the dispersion relation (2). We seek for this particular wave number the value of the spatial amplification rate $\tilde{k}_{i}$ such that $\omega\left(\tilde{k}_{r}\right.$ $+i \widetilde{k}_{i}$ ) denoted $\omega_{S}$ is real. For small amplification rates, we make a Taylor expansion of $\omega_{S}$ for $\tilde{k}_{i}$ small. If we suppose that $\omega$ is an analytical function of $k$, the Cauchy-Riemann relation holds and the Taylor expansion becomes

$\omega_{S}=\omega_{r}\left(\tilde{k}_{r}\right)+i \omega_{i}\left(\tilde{k}_{r}\right)-\tilde{k}_{i} \frac{\partial \omega_{i}}{\partial k_{r}}\left(\tilde{k}_{r}\right)+i \tilde{k}_{i} \frac{\partial \omega_{r}}{\partial k_{r}}\left(\tilde{k}_{r}\right)+O\left(\tilde{k}_{i}^{2}\right)$.

We conclude that for $\widetilde{k}_{i}\left(\partial \omega_{i} / \partial k_{r}\right)\left(\widetilde{k}_{r}\right)$ small, the frequency associated with $\tilde{k}_{r}$ is the same in the temporal case $\left(\widetilde{k}_{i}=0\right)$ and in the spatial case $\left[\operatorname{Im}\left(\omega_{s}\right)=0\right]$

$$
\omega_{S} \sim \omega_{r}\left(\tilde{k}_{r}\right) .
$$

Moreover, since spatial mode corresponds to find $\widetilde{k}_{i}$ such that $\omega_{s}$ is real, we obtain, except if the group velocity $\partial \omega_{r} / \partial k_{r}\left(\tilde{k}_{r}\right)$ vanishes

$$
\tilde{k}_{i}=-\frac{\omega_{i}\left(\tilde{k}_{r}\right)}{\frac{\partial \omega_{r}}{\partial k_{r}}\left(\tilde{k}_{r}\right)} .
$$

For $R_{i}=4$, the temporal amplification rate $\omega_{i}$ has been plotted on Fig. 11(a). The spatial amplification rate obtained from Gaster transformation $\tilde{k}_{i}$ is computed by (32) for each value of the wave number. For this value, the frequency $\omega_{S}$ associated to the spatial mode is obtained from the temporal theory (31). For $a$ varying from 0.2 to 1.5 , we plot as a dotted curve the spatial amplification rates thus obtained as a function of $\omega_{S}$ for the right-going mode (Fig. 13) and for the left-going mode (Fig. 14).

For the right-going Holmboe mode represented on Fig. 13 , the dotted branches obtained from (31) and (32) fit very 
well the spatial branches obtained numerically (in solid curve). For the left-going Holmboe mode far from the convective-absolute instability thresholds $a=0.438$ and $a$ $=1$, the Gaster transformation (32) gives a good prediction of the spatial amplification rate. Near the two thresholds, $a$ $=0.43$ and $a=1.01$, the solid and dotted curves are very different. The group velocities are very small, the spatial growth rate is large and the Taylor expansion (30) is no longer valid since terms of order $k_{i}^{2}$ can no longer be neglected. Close to $a=1$, the failure is also imputable to the nonanalyticity of the dispersion relation near the branch cut where the "pinching at infinity" occurs. Cauchy-Riemann relations do not hold, and so the expansion given in (30) is no longer valid. We conclude that, except close to the absolute-convective thresholds, the Gaster transformation predicts remarkably well the spatial instability.

In Exchange flows, the two layers flow in opposite directions. Such flows are characterized in our model (Fig. 1) by a mean advection $a$ between 0 and 1 (by convention $a$ is positive). When the Richardson number is lower than 0.07, exchange flows are absolutely unstable in the whole range [0,1[ (Fig. 4), and are able to exhibit self-sustained oscillations. In contrast when $R_{i}$ is larger than 0.07 , exchange flows are absolutely unstable for mean advection larger than $a_{c}\left(R_{i}\right)$ and once again self-sustained oscillations may occur. For $a$ smaller than $a_{c}\left(R_{i}\right)$, the flow is convectively unstable, but since both upstream and downstream propagating spatial branches are unstable, a self-sustained resonance may be easily triggered by reflective boundary conditions and the dynamics of exchange flow deserve further analysis.

Wedge flows are a particular case of exchange flows, with one layer arrested. In our model (Fig. 1), they correspond to $a=1$. These flows are marginal since the transition from absolute to convective instability occurs at $a=1$ for all values of $R_{i}$. This result is not an artifact induced by the singularity of the broken line velocity profile used to model the flow. Indeed, Balsa ${ }^{38}$ has shown numerically for $R_{i}=0$, that a smoothing of the velocity profile imposed at the edges of the shear layer near $y= \pm 1$ (Fig. 1) does not modify this absolute-convective transition. Of course, strong changes in the velocity profile used to model the shear are known to modify the threshold. As an example, in the case of a homogeneous hyperbolic tangent velocity profile, the theoretical study carried out by Huerre and Monkewitz ${ }^{31}$ for homogeneous mixing-layers $\left(R_{i}=0\right)$ has revealed that the threshold value is $a=0.760$ instead of $a=1$. This explains why Pawlak and $\mathrm{Armi},{ }^{22}$ investigating linear spatial instability in the case of Boussinesq approximation, for a flow modeled by hyperbolic tangent velocity and density profiles with different thickness characteristics, found that the instability was convective at $a=0.89$. Nevertheless, in their experiments, Pawlak and $\mathrm{Armi}^{22}$ put forward a very regular regime which consists of a first roll up of the interface as in the homogeneous case. This first vortex core separates from the vorticity source and a second core develops which pairs with the first one. This mechanism, called "leapfrog pairing,",22 persists into fully developed regions and might be the signature of a self-sustained mode (see Brancher and $\mathrm{Chomaz}^{39}$ for a discussion in the case $R_{i}=0$ ). Further experiments are needed to determine whether or not self-sustained oscillations are observed in exchange flows.

In Mixing-layers, the two layers flow in the same directions. Such flows are characterized in our model (Fig. 1) by a mean advection $a$ larger than unity. Whatever the stratification, unstable mixing-layers are convectively unstable (Fig. 4). Convectively unstable flows are well known to be extremely sensitive to external forcing. Figures 9(c) and 9(d) have shown that the two Holmboe modes may be well separated in frequency for a particular value of the Richardson number. We believe that this frequency selection mechanism may explain the observations of Browand and Wang ${ }^{17}$ in a stratified channel. They demonstrate that depending on the activation or not to an harmonic forcing on the splitter plate separating the two layers of fluids, one or two traveling waves were observed. At finite amplitude, the two Holmboe waves which form cusps in the upper and the lower layer ${ }^{10}$ are not always observed experimentally. Koop and Browand, ${ }^{11}$ in their experiment, introduced dye separately in the upper and lower layer. They reported a rolling up of dye, indicating a concentration of vorticity, only in the layer having the smaller velocity (the upper one in their experiment) and a cusp of the interface only in this layer. This phenomenon was called "one-sidedness" by Maxworthy and Browand. ${ }^{12}$ Recently, Zhu and Lawrence, ${ }^{40}$ in exchange flows apparatus, obtained the experimental evidence of both Holmboe waves (the two traveling waves) and one-sided Holmboe wave (one traveling wave). One-sided waves arise from the result of a loss of symmetry of the flow. For Haigh and Lawrence, ${ }^{41}$ there are two ways for the background flow to lose its symmetry: either by displacing the density interface with respect to the center of the shear layer or by having horizontal boundaries placed at different distances from the center of the shear layer. ${ }^{41}$ Whereas this is true for temporal theory or numerical experiments in periodic boxes when the symmetry is unbroken, in the spatial theory or in real experiments as soon as $a$ is not zero, the symmetry is broken by the inlet condition. The frequency of the Holmboe wave developing in the slower layer is smaller and its spatial growth rate is larger than its symmetric counterpart as shown on Fig. 9. If a white noise is imposed at the inlet then, as in Koop and Browand's experiments, ${ }^{11}$ the Holmboe mode propagating with the slower stream should dominate the evolution. If the flow is forced by a wave-maker or by an external noise for example at a particular frequency, it seems possible to favor the growth of the other mode since they are unstable to different frequency ranges.

\section{ACKNOWLEDGMENTS}

The authors are grateful to G. Pawlak, L. Armi, P. Huerre, Olivier Pouliquen, and Burt Tilley for helpful discussions. Special thanks go to L. Tuckerman for her careful reading of the manuscript.

${ }^{1}$ L. Armi and D. M. Farmer, "The flow of Mediterranean water through the Strait of Gibraltar," Prog. Oceanogr. 21, 1 (1988).

${ }^{2}$ G. Pawlak and L. Armi, "Hydraulics of two-layer arrested wedge flows," J. Hydraul. Res. 35, 603 (1997). 
${ }^{3}$ D. M. Farmer and H. Freeland, "The physical oceanography of fjords," Prog. Oceanogr. 12, 147 (1983).

${ }^{4}$ P. Pettre and J. C. Andre, "Surface-pressure change through Loewe's phenomena and katabatic flow jumps. Study of two cases in Adelie Land Antartica," J. Atmos. Sci. 48, 557 (1991).

${ }^{5}$ J. E. Simpson, Gravity Currents: In the Environment and the Laboratory (Ellis Horwood, Chichester, 1987).

${ }^{6} \mathrm{~J}$. W. Miles, "On the stability of heterogeneous shear flow," J. Fluid Mech. 10, 496 (1961)

${ }^{7}$ L. N. Howard, "Note on a paper of John W. Miles," J. Fluid Mech. 10, 509 (1961)

${ }^{8}$ C. S. Yih, "Stability of and waves in stratified flows," Proceedings of Symposium Naval Hydrodynamics, 8th, Pasadena, California (1970).

${ }^{9}$ L. N. Howard and S. A. Maslowe, "Stability of stratified shear flows," Boundary-Layer Meteorol. 4(10), 511 (1973).

${ }^{10}$ F. K. Browand and C. D. Winant, "Laboratory observations of shear layer instability in a stratified flow," Boundary-Layer Meteorol. 5, 67 (1973).

${ }^{11}$ C. G. Koop and F. K. Browand, "Instability and turbulence in a stratified fluid with shear," J. Fluid Mech. 93, 135 (1979).

${ }^{12}$ T. Maxworthy and F. K. Browand, "Experiments in rotating and stratified flows: Oceanographic application," Annu. Rev. 7, 273 (1975).

${ }^{13}$ P. Hazel, "Numerical studies of the stability of inviscid stratified shear flows," J. Fluid Mech. 51, 39 (1972).

${ }^{14}$ W. D. Smyth and W. R. Peltier, "The transition between KelvinHelmholtz and Holmboe instability: An investigation of the overreflection hypothesis," J. Atmos. Sci. 46, 2698 (1989).

${ }^{15}$ G. A. Lawrence, F. K. Browand, and L. G. Redekopp, "The stability of a sheared density interface," Phys. Fluids A 3, 2360 (1991).

${ }^{16} \mathrm{~J}$. Holmboe, "On the behaviour of symmetric waves in stratified shear flows," Geofys. Publ. 24, 67 (1962).

${ }^{17}$ F. K. Browand and Y. H. Wang, "An experiment on the growth of small disturbances at the interface between two streams of different densities and velocities," Proceedings of the International Symposium on Stratified Flows, August 29-31, Novosibirsk, Soviet Union (1972).

${ }^{18}$ W. D. Smyth, G. P. Klassen, and W. R. Peltier, "Finite amplitude Holmboe waves," Geophys. Astrophys. Fluid Dyn. 43, 181 (1988).

${ }^{19}$ P. Huerre and P. A. Monkewitz, "Local and global instabilities in spatially developing flows," Annu. Rev. Fluid Mech. 22, 473 (1990).

${ }^{20}$ S. J. Lin and R. T. Pierrehumbert, "Absolute and convective instability of inviscid stratified shear flows," in Proceedings of the 4th International Symposium of Stratified Flow, Caltech (Elsevier, New York, 1987).

${ }^{21}$ G. S. Triantafyllou "Note on the Kelvin-Helmholtz instability of stratified fluids," Phys. Fluids 6, 164 (1994).

${ }^{22}$ G. Pawlak and L. Armi, "Vortex dynamics in a spatially accelerating shear layer," J. Fluid Mech. 376, 1 (1999).
${ }^{23}$ P. Kolodner, C. M. Surko, and H. Williams, "Dynamics of traveling waves near the onset of convection in binary fluid mixtures," Physica D 37, 319 (1989).

${ }^{24}$ P. G. Drazin and W. H. Reid, Hydrodynamic Stability (Cambridge University Press, Cambridge, 1981).

${ }^{25}$ C. S. Yih, "Stability of two-dimensional parallel flows for threedimensional disturbances," Q. Appl. Math. 12, 434 (1955).

${ }^{26}$ O. Pouliquen, J. M. Chomaz, and P. Huerre, "Propagating Holmboe waves at the interface between two immiscible fluids," J. Fluid Mech. 266, 277 (1994).

${ }^{27}$ S. A. Thorpe, "Experiments on the instability of stratified shear flows: Immiscible fluids," J. Fluid Mech. 39, 25 (1969).

${ }^{28}$ Lord Rayleigh, Theory of Sound (Dover, New York, 1955).

${ }^{29}$ R. J. Briggs, Electron-Stream Interaction with Plasmas, Research Monograph (MIT Press, Cambridge, MA. 1964), Vol. 29.

${ }^{30}$ A. Bers, "Linear waves and instabilities," Physique des Plasmas, edited by C. Dewitt and J. Peyraud (Gordon and Breach, New York, 1975).

${ }^{31}$ P. Huerre and P. A. Monkewitz, "Absolute and convective instability in free shear layers," J. Fluid Mech. 159, 151 (1985).

${ }^{32} \mathrm{~J}$. T Balsa, "On the receptivity of free shear layers to two-dimensional external excitation," J. Fluid Mech. 187, 155 (1988).

${ }^{33}$ D. Bechert, "Uber mehrfache und stromauf Laufende wellen," in Freistrahlen DFVLR Rep. DLR-FBN-72-06.

${ }^{34}$ J. M. Chomaz, "Linear and non-linear, local and global stability analysis of open flows," Comptes-rendus de l'Ecole des Houches Turbulence in Spatially Extended Systems (Springer-Verlag, Berlin, 1992).

${ }^{35}$ T. Loiseleux, J. M Chomaz, and P. Huerre, "The effect of swirl on jets and wakes: Linear instability of the Rankine vortex with axial flow," Phys. Fluids 10, 1120 (1998).

${ }^{36} \mathrm{M}$. Gaster, "A note on the relation between temporally increasing and spatially increasing disturbances in hydrodynamic stability," J. Fluid Mech. 14, 222 (1964)

${ }^{37}$ C. P. Caulfield, "Multiple linear instability of layered stratified shear flow,” J. Fluid Mech. 258, 255 (1994).

${ }^{38}$ J. T. Balsa, "On the spatial instability of piecewise linear free shear layers," J. Fluid Mech. 174, 553 (1987).

${ }^{39} \mathrm{P}$. Brancher and J. M. Chomaz, "Absolute and convective instabilities in spatially periodic shear flows," Phys. Rev. Lett. 78, 658 (1997).

${ }^{40}$ D. Z. Zhu and G. A. Lawrence, "Holmboe's instability in exchange flows," J. Fluid Mech. 429, 391 (2001).

${ }^{41}$ S. P. Haigh and G. A. Lawrence, "Symmetric and nonsymmetric Holmboe instabilities in an inviscid flow," Phys. Fluids 11, 1459 (1999). 